\title{
Pioglitazone and Risk of Chronic Obstructive Pulmonary Disease in Patients with Type 2 Diabetes Mellitus: A Retrospective Cohort Study
}

\author{
Chin-Hsiao Tseng $\mathbb{( D D}^{1-3}$ \\ Taiwan \\ Correspondence: Chin-Hsiao Tseng, Tel/Fax +886 22388 3578, Email ccktsh@ms6.hinet.net
}

'Department of Internal Medicine, National Taiwan University College of Medicine, Taipei, Taiwan; ${ }^{2}$ Division of Endocrinology and Metabolism, Department of Internal Medicine, National Taiwan University Hospital, Taipei, Taiwan; ${ }^{3}$ National Institute of Environmental Health Sciences, Zhunan,

Background: Pioglitazone's effect on chronic obstructive pulmonary disease (COPD) has rarely been studied.

Purpose: This retrospective observational study investigated whether the use of pioglitazone would affect the risk of COPD in patients with type 2 diabetes mellitus.

Patients and Methods: The Taiwan's National Health Insurance database was used to enroll 9487 matched pairs of ever users and never users of pioglitazone based on propensity score from a cohort of 350,536 patients. The enrolled patients had a new diagnosis of type 2 diabetes mellitus between 1999 and 2008 and were not having a diagnosis of COPD before January 1, 2009. They were then followed up for COPD, starting from January 1, 2009 until December 31, 2011. Diagnosis of COPD was based on the codes of 491 for chronic bronchitis and 492 for emphysema based on the International Classification of Diseases, Ninth Revision, Clinical Modification. Cox regression was used to estimate hazard ratios. The interactions between pioglitazone and COPD risk factors including pneumonia, pulmonary tuberculosis and tobacco abuse were also investigated.

Results: In 9487 never users and 9487 ever users of pioglitazone, the case numbers of incident COPD were 359 and 295 , respectively. The respective incidence rates of COPD were 1484.73 and 1167.61 per 100,000 person-years. The overall hazard ratio (95\% confidence interval) for COPD that compared ever to never users was 0.778 (0.667-0.908). The hazard ratios for the tertiles of cumulative duration of pioglitazone therapy (cutoffs: $<11.0,11.0-19.6$ and $>19.6$ months) to never users were 0.904 (0.729-1.121), $0.727(0.578-0.914)$ and $0.715(0.570-0.896)$, respectively. No interactions between pioglitazone and COPD risk factors including pneumonia, pulmonary tuberculosis and tobacco abuse were noted.

Conclusion: Pioglitazone use is associated with a significantly lower risk of COPD.

Keywords: chronic bronchitis, emphysema, pharmacoepidemiological study, risk factors, Taiwan, thiazolidinediones

\section{Introduction}

Patients with type 2 diabetes mellitus (T2DM) are characterized by insulin resistance in the early stage of the disease. However, they can have impaired insulin secretion because of pancreatic $\beta$-cell failure in later stages. Patients with T2DM develop hyperglycemia and have a significantly higher risk of hypertension and dyslipidemia. Various acute and chronic vascular complications may set in if the metabolic dysfunctions are not appropriately corrected or controlled. Hyperglycemia triggers a pro-inflammatory status and oxidative stress in the bodies, which predispose to the development of cancer, ${ }^{1}$ aging, ${ }^{2}$ atherosclerosis, ${ }^{2}$ kidney disease, ${ }^{2}$ dementia, ${ }^{3}$ osteoarthritis, ${ }^{4}$ muscle wasting ${ }^{4}$ and chronic obstructive pulmonary disease (COPD). ${ }^{4,5}$

The pathogenetic link between T2DM and COPD may involve pro-inflammatory status, microangiopathy, glycosylation of proteins in the lung and the bronchial trees, increased risk of respiratory infection, thickening of pulmonary basal lamina and increased responsiveness of airway smooth muscle cells to contractile agents under high glucose concentrations. ${ }^{5}$ 


\section{Graphical Abstract}

\section{Cohort}

Taiwan NHI database

New diabetes patients without COPD in 1999-2008 $(\mathrm{N}=350,536)$

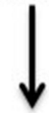

\section{Matched samples}

9487 never users of pioglitazone

9487 ever users of pioglitazone

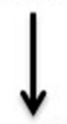

Follow-up

2009 to 2011

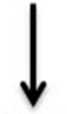

Outcome

COPD

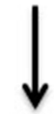

Findings

Pioglitazone Yes versus No: COPD risk reduces $22 \%$

Thiazolidinediones (TZDs) are a class of oral antidiabetic drugs that target the peroxisome proliferator-activator receptor gamma (PPAR $\gamma)$ in adipocytes. ${ }^{6}$ They lower circulating free fatty acids and restrict the availability of lipids to liver and muscle, resulting in the improvement of insulin resistance. ${ }^{6}$ In Taiwan, only rosiglitazone and pioglitazone have been approved for clinical use. ${ }^{7}$ In 2007, a meta-analysis suggested a potential risk of myocardial infarction and cardiovascular death associated with rosiglitazone use. ${ }^{8}$ Since then, rosiglitazone has been withdrawn from the market or its use has been discontinued in many countries including Taiwan. In contrary to rosiglitazone, pioglitazone significantly improves lipid profiles ${ }^{9}$ and reduces the risk of cardiovascular diseases in patients with $\mathrm{T}_{2} \mathrm{DM}^{10}$ or in patients with ischemic stroke and insulin resistance. ${ }^{11}$ Although a potential risk of bladder cancer has been raised since 2011 in the interim analysis of the Kaiser Permanente Northern California study, ${ }^{12}$ this issue has been controversial without a conclusive result according to the latest assessment by the Food and Drug Administration of the USA. ${ }^{13}$ Our previous studies that analyzed the reimbursement database of the Taiwan's National Health Insurance (NHI) suggested a null association between bladder cancer and TZDs, either pioglitazone ${ }^{14,15}$ or rosiglitazone. ${ }^{16}$ Pioglitazone survives the market and is currently the only TZD available for clinical use in Taiwan.

Pioglitazone has anti-inflammatory effects and has been shown to inhibit the release of pro-inflammatory cytokines and mucus production in the lung. ${ }^{5}$ In our previous study, although an overall neutral effect was found between pioglitazone use and lung cancer (hazard ratio: $0.925,95 \%$ confidence interval: $0.784-1.091$ ), the risk was significantly reduced in patients who had a cumulative dose of pioglitazone $>15,300 \mathrm{mg}$ (hazard ratio: $0.498,95 \%$ confidence interval $0.379-0.653, P<$ 0.0001). ${ }^{17}$ Because COPD is a well-recognized risk factor for lung cancer, ${ }^{18}$ one of the possible explanations for the reduced risk of lung cancer associated with prolonged pioglitazone use is through an effect on the reduction of COPD. 
Pioglitazone effectively prevents sepsis-induced lung injury in mice by reducing inflammation. ${ }^{19}$ In emphysema mouse models, pioglitazone-pretreated adipose-derived mesenchymal stem cells ${ }^{20}$ and Wharton's Jelly-derived mesenchymal stem cells ${ }^{21}$ were more potent in the repair of lung injury. In in vitro and in vivo models of COPD, TZDs, including rosiglitazone, pioglitazone and ciglitazone, attenuated pulmonary inflammation. ${ }^{22,23}$ In humans, Rinne et al conducted a retrospective cohort analysis in the US veterans and showed that TZDs (mainly rosiglitazone)-treated diabetes patients with COPD (only $2 \%$ of them were treated with pioglitazone) might have a reduced exacerbation of the disease. ${ }^{24}$ To our knowledge, whether pioglitazone might affect the risk of COPD has rarely been researched in human epidemiological studies. Therefore, we investigated whether pioglitazone use in patients with T2DM might affect the risk of COPD.

\section{Patients and Methods}

This is a retrospective cohort study based on propensity score (PS)-matched pairs of pioglitazone ever users and never users derived from the reimbursement database of the NHI. The NHI is a compulsory and unique healthcare system implemented since March 1, 1995 in Taiwan. It covers $>99.6 \%$ of the Taiwan's population. All in-hospitals and more than $93 \%$ of all medical settings in Taiwan have contracts with the Bureau of the NHI to provide medical care to the insurants. Computerized medical records including disease diagnoses, medication prescriptions and performed procedures have to be submitted to the Bureau of the NHI for reimbursement purposes. Academic research using the database is allowed if the submitted research proposal is approved by an institutional review board. This study was approved by the Research Ethics Committee of the National Health Research Institutes (number 99274). According to local regulations, informed consent is not required because all personal information needs to be de-identified before the database is released.

Throughout the research period, the disease coding system was the International Classification of Diseases, Ninth Revision, Clinical Modification (ICD-9-CM). Accordingly, diabetes mellitus was coded 250.XX and the codes of 491 (chronic bronchitis) and 492 (emphysema) were used to define COPD in the study.

Figure 1 illustrates the step-by-step procedures in creating the PS-matched pairs of ever users and never users of pioglitazone enrolled for analyses in the study. First, 350,536 patients newly diagnosed of diabetes mellitus from 1999 to

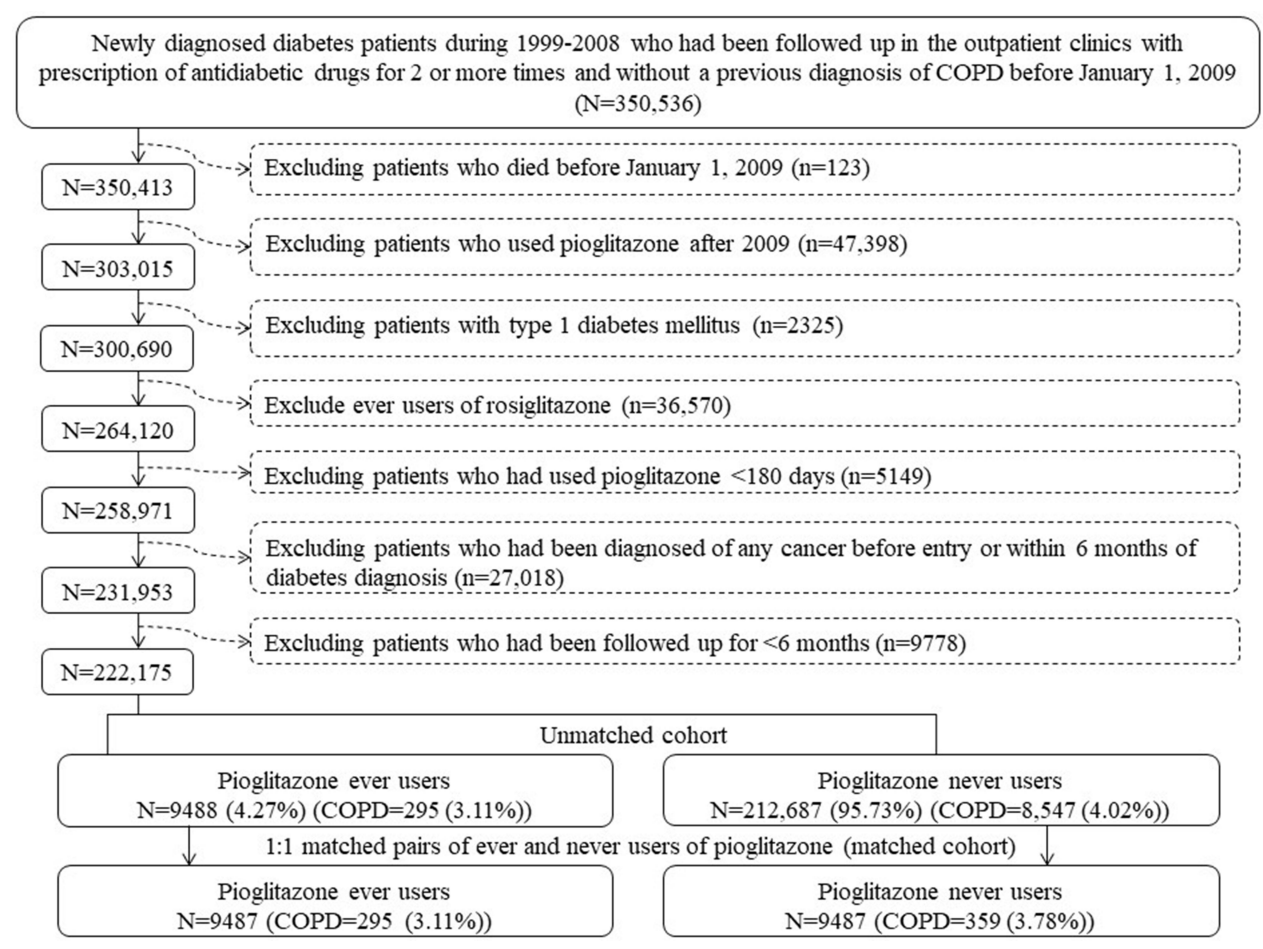

Figure I The step-by-step procedures applied in the enrollment of propensity score-matched pairs of ever users and never users of pioglitazone. Abbreviation: COPD, chronic obstructive pulmonary disease. 
2008 with prescription of antidiabetic drugs for at least two times and without a previous diagnosis of COPD before January 1 , 2009 were identified from the outpatient clinics. To ensure a new onset of diabetes mellitus after 1999, the patients should not have a previous diagnosis of diabetes mellitus from 1996 to 1998. The following ineligible patients were then excluded: 1) patients who died before January 1, $2009(\mathrm{n}=123), 2)$ patients who had a first prescription of pioglitazone after $2009(\mathrm{n}=$ 47,398), 3) patients with a diagnosis of type 1 diabetes mellitus $(\mathrm{n}=2325), 4)$ patients who had ever been prescribed rosiglitazone $(\mathrm{n}=36,570), 5)$ patients who had used pioglitazone for less than 180 days $(\mathrm{n}=5149), 6)$ patients who had been diagnosed of cancer before entry or within six months after diabetes diagnosis $(n=27,018$, the lifespan of cancer patients is expected to be shortened and therefore inclusion of these patients might distort the calculation of person-years in the follow-up time; furthermore, lung metastasis of cancer might have been misdiagnosed as COPD), and 7) patients who had a short followup duration of $<180$ days $(\mathrm{n}=9778)$. We identified 9488 ever users and 212,687 never users of pioglitazone in the unmatched cohort. PS for pioglitazone treatment was generated by logistic regression from independent variables including all those shown in Table 1 . The Greedy $8 \rightarrow 1$ digit match algorithm ${ }^{25}$ was applied to create a cohort of PS-matched pairs of ever users and never users of pioglitazone. As a result, 9487 pairs were successfully matched.

Cumulative duration of pioglitazone therapy in months was calculated as an indicator of a possible dose-response relationship. The following categories of variables were retrieved from the database as potential confounders: basic data, major comorbidities associated with diabetes mellitus, diabetes-related complications, factors that might affect exposure/ outcome, antidiabetic drugs and medications commonly prescribed to diabetes patients or drugs that might affect exposure/outcome. The variables of basic data included age, sex, diabetes duration, occupation and living region. Living regions were classified according to geographical distribution into Taipei, Northern, Central, Southern, and KaoPing/Eastern. Occupation was classified as class I (civil servants, teachers, employees of governmental or private businesses, professionals and technicians), class II (people without a specific employer, self-employed people or seamen), class III (farmers or fishermen) and class IV (low-income families supported by social welfare, or veterans).

Major comorbidities included the diagnoses of hypertension, dyslipidemia and obesity. Diabetes-related complications retrieved from the database included nephropathy, eye disease, diabetic polyneuropathy, stroke, ischemic heart disease and peripheral arterial disease. The following diagnoses were considered as factors that might affect exposure/ outcome: head injury, Parkinson's disease, hypoglycemia, tobacco abuse, alcohol-related diagnoses, heart failure, gingival and periodontal diseases, pneumonia, pulmonary tuberculosis, osteoporosis and human immunodeficiency virus infection. The ICD-9-CM codes for these diagnoses have been reported previously. ${ }^{26,27}$

Antidiabetic drugs included insulin, sulfonylureas, metformin, meglitinide and acarbose. Medications commonly prescribed to diabetes patients or drugs that might affect exposure/outcome included angiotensin converting enzyme inhibitors/angiotensin receptor blockers, calcium channel blockers, statins, fibrates, aspirin and corticosteroids (consistent use $\geq 90$ days).

Standardized difference, which is less affected by sample sizes as observed in statistical tests, was calculated for each of the variables, as recommended by Austin and Stuart. ${ }^{28}$ There is no consensus as to the threshold value of standardized difference for an indication of confounding, but many investigators suggested a threshold value of $>10 \%$.

Incidence density of COPD was calculated for various subgroups of patients categorized according to the exposure to pioglitazone, ie, never users, ever users and each of the tertiles of cumulative duration of pioglitazone therapy. The incidence numerator was the case number of COPD newly diagnosed during follow-up, and the incidence denominator was the person-years of follow-up. All patients were followed up starting on January 1, 2009 and the follow-up duration ended at a time up to December 31, 2011 until whichever of the following events occurred first: a COPD diagnosis, the last reimbursement record or the date of death.

Kaplan-Meier curves for COPD-free probability were plotted with regard to pioglitazone exposure. We compared 1) ever users to never users; and 2) the tertile cutoffs of cumulative duration of pioglitazone therapy to never users. Log rank test was used to examine whether the differences among different subgroups were statistically significant.

Cox regression was conducted to estimate adjusted hazard ratios and their $95 \%$ confidence intervals that compared ever users to never users or compared patients categorized in each tertile of the cumulative duration of pioglitazone therapy to never users. All variables listed in Table 1 were entered as independent variables for adjustment. 
Table I Comparisons of Characteristics Between Pioglitazone Never Users and Ever Users

\begin{tabular}{|c|c|c|c|c|c|}
\hline \multirow[t]{2}{*}{ Characteristics } & \multicolumn{2}{|c|}{$\begin{array}{l}\text { Never Users } \\
(n=9487)\end{array}$} & \multicolumn{2}{|c|}{$\begin{array}{l}\text { Ever Users } \\
(n=9487)\end{array}$} & \multirow[t]{2}{*}{ Standardized Difference } \\
\hline & $\mathbf{n}$ & $\%$ & $\mathbf{n}$ & $\%$ & \\
\hline \multicolumn{6}{|l|}{ Basic data } \\
\hline Age (years) & 59.44 & 11.82 & 59.41 & 11.19 & -0.26 \\
\hline Sex (men) & 5426 & 57.19 & 5387 & 56.78 & -0.88 \\
\hline Diabetes duration (years) & 6.38 & 2.81 & 6.37 & 2.60 & -0.29 \\
\hline \multicolumn{6}{|l|}{ Occupation } \\
\hline 1 & 3823 & 40.30 & 3834 & 40.41 & \\
\hline II & 2208 & 23.27 & 2211 & 23.31 & 0.12 \\
\hline III & 1787 & 18.84 & 1732 & 18.26 & -1.40 \\
\hline IV & 1669 & 17.59 & 1710 & 18.02 & 1.26 \\
\hline \multicolumn{6}{|l|}{ Living region } \\
\hline Taipei & 3831 & 40.38 & 3812 & 40.18 & \\
\hline Northern & 931 & 9.81 & 997 & $|0.5|$ & 2.33 \\
\hline Central & 1523 & 16.05 & 1483 & 15.63 & -1.05 \\
\hline Southern & 1156 & 12.19 & 1142 & 12.04 & -0.40 \\
\hline Kao-Ping and Eastern & 2046 & 21.57 & 2053 & 21.64 & 0.24 \\
\hline \multicolumn{6}{|c|}{ Major comorbidities associated with diabetes mellitus } \\
\hline Hypertension & 7576 & 79.86 & 7536 & 79.44 & -1.06 \\
\hline Dyslipidemia & 8107 & 85.45 & 8073 & 85.10 & -0.96 \\
\hline Obesity & 529 & 5.58 & 597 & 6.29 & 2.89 \\
\hline \multicolumn{6}{|c|}{ Diabetes-related complications } \\
\hline Nephropathy & 2258 & 23.80 & 2174 & 22.92 & -2.22 \\
\hline Eye disease & 3151 & 33.21 & 3183 & 33.55 & 0.67 \\
\hline Diabetic polyneuropathy & 2402 & 25.32 & 2487 & 26.21 & 2.17 \\
\hline Stroke & 2049 & 21.60 & 2032 & 21.42 & -0.28 \\
\hline Ischemic heart disease & 3653 & 38.51 & 3618 & 38.14 & -0.70 \\
\hline Peripheral arterial disease & 2135 & 22.50 & 2074 & 21.86 & -1.54 \\
\hline \multicolumn{6}{|c|}{ Factors that might affect exposure/outcome } \\
\hline Head injury & 312 & 3.29 & 286 & 3.01 & -1.61 \\
\hline Parkinson's disease & 148 & 1.56 & 144 & 1.52 & -0.20 \\
\hline Hypoglycemia & 299 & 3.15 & 302 & 3.18 & 0.16 \\
\hline Tobacco abuse & 338 & 3.56 & 325 & 3.43 & -0.79 \\
\hline Alcohol-related diagnoses & 477 & 5.03 & 479 & 5.05 & 0.19 \\
\hline
\end{tabular}


Table I (Continued).

\begin{tabular}{|c|c|c|c|c|c|}
\hline \multirow[t]{2}{*}{ Characteristics } & \multicolumn{2}{|c|}{$\begin{array}{c}\text { Never Users } \\
(n=9487)\end{array}$} & \multicolumn{2}{|c|}{$\begin{array}{c}\text { Ever Users } \\
(n=9487)\end{array}$} & \multirow[t]{2}{*}{ Standardized Difference } \\
\hline & $\mathbf{n}$ & $\%$ & $\mathbf{n}$ & $\%$ & \\
\hline Heart failure & 1158 & 12.21 & 1137 & 11.98 & -0.63 \\
\hline Gingival and periodontal diseases & 8312 & 87.61 & 8316 & 87.66 & 0.11 \\
\hline Pneumonia & 801 & 8.44 & 843 & 8.89 & 1.49 \\
\hline Pulmonary tuberculosis & 182 & 1.92 & 178 & 1.88 & -0.39 \\
\hline Osteoporosis & $|32|$ & 13.92 & 1348 & 14.21 & 0.86 \\
\hline Human immunodeficiency virus infection & 13 & 0.14 & 4 & 0.04 & $-4.7 \mathrm{I}$ \\
\hline \multicolumn{6}{|l|}{ Antidiabetic drugs } \\
\hline Insulin & 269 & 2.84 & 291 & 3.07 & 1.39 \\
\hline Sulfonylureas & 6638 & 69.97 & 6650 & 70.10 & 0.46 \\
\hline Metformin & 7202 & 75.91 & 7129 & 75.14 & -1.93 \\
\hline Meglitinide & 625 & 6.59 & 638 & 6.72 & 0.56 \\
\hline Acarbose & 1286 & 13.56 & 1282 & 13.51 & -0.07 \\
\hline \multicolumn{6}{|c|}{ Medications commonly prescribed to diabetes patients or drugs that might affect exposure/outcome } \\
\hline Angiotensin converting enzyme inhibitors/Angiotensin receptor blockers & 6869 & 72.40 & 6880 & 72.52 & 0.14 \\
\hline Calcium channel blockers & 5058 & 53.32 & 5079 & 53.54 & 0.54 \\
\hline Statins & 7077 & 74.60 & 7083 & 74.66 & 0.16 \\
\hline Fibrates & 4154 & 43.79 & 4147 & 43.71 & -0.21 \\
\hline Aspirin & 5377 & 56.68 & 5328 & 56.16 & -1.00 \\
\hline Corticosteroids & 187 & 1.97 & 166 & 1.75 & -1.62 \\
\hline
\end{tabular}

Note: Age and diabetes duration are expressed as mean and standard deviation.

The joint effects of pioglitazone and major risk factors of COPD (ie, pneumonia, pulmonary tuberculosis and tobacco abuse) were then evaluated by estimating the hazard ratios for COPD in the following subgroups, considering the presence and absence of COPD risk factors and pioglitazone use: 1) COPD risk factor (+)/pioglitazone (-) as the referent group; 2) COPD risk factor $(+) /$ pioglitazone $(+)$; 3) COPD risk factor (-)/pioglitazone (-); and 4) COPD risk factor $(-) /$ pioglitazone $(+)$. Additionally, the values of $P$-trend and $P$-interaction were derived from the models.

SAS statistical software (version 9.4, SAS Institute, Cary, NC) was used. Statistical significance was denoted by a $P$-value $<0.05$.

\section{Results}

Table 1 compares the characteristics between pioglitazone never users and ever users. The values of standardized difference for all variables were $<10 \%$, suggesting that a well-matched cohort of ever and never users of pioglitazone had been created from the matching procedure.

Figure 2 shows the Kaplan-Meier curves. The curves that compare ever users to never users in Figure 2A indicated a lower risk of COPD in ever users $(P=0.0014)$. The curves in Figure 2B for never users and the tertile cutoffs of 
A

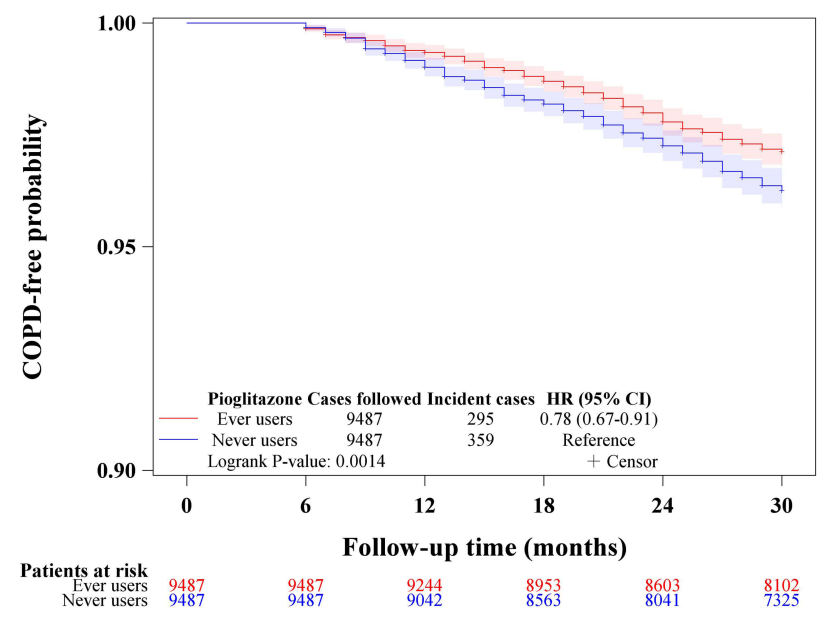

B

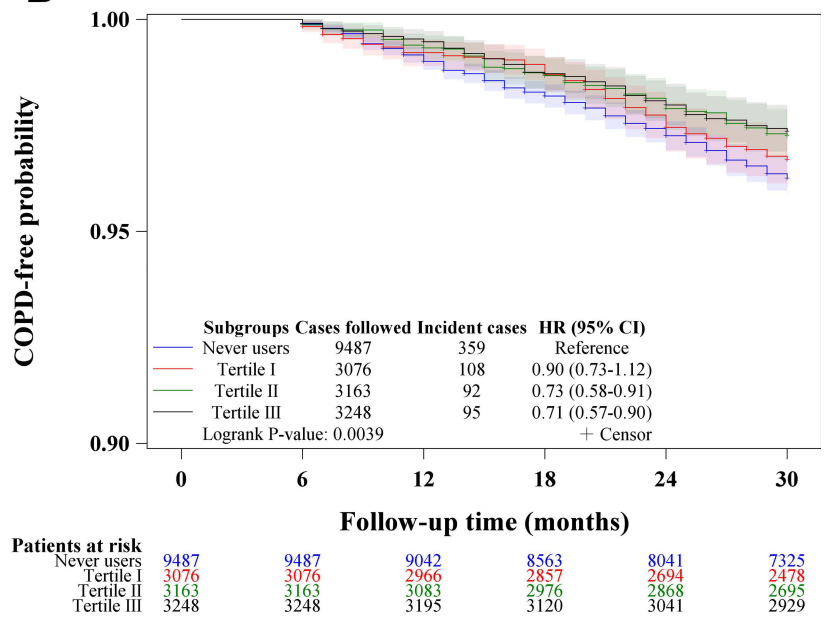

Figure 2 The Kaplan-Meier curves for COPD-free probability with regards to pioglitazone exposure. $(\mathbf{A})$ compares pioglitazone ever users to never users (logrank test, $P=$ 0.00I4). (B) compares tertiles of cumulative duration of pioglitazone therapy to never users (logrank test, $P=0.0039$ ).

Abbreviation: COPD, chronic obstructive pulmonary disease.

cumulative duration of pioglitazone therapy suggested a dose-dependent reduction of COPD risk in corresponding to increasing cumulative duration of pioglitazone therapy $(P=0.0039)$.

Table 2 shows the incidence rates of COPD for different subgroups of pioglitazone exposure and the hazard ratios comparing exposed patients (ever users and users in each of the tertiles of cumulative duration) to unexposed patients. The overall hazard ratios suggested that pioglitazone use was associated with a significantly $22 \%$ risk reduction of COPD (hazard ratio: $0.778,95 \%$ confidence interval: $0.667-0.908$ ). Tertile analysis shows a risk reduction in the second and third tertiles when cumulative duration of pioglitazone therapy has reached 11 months or more.

Table 3 shows the joint effects of pioglitazone and the COPD risk factors. The hazard ratios were lowest in patients who did not have the risk factors and had been treated with pioglitazone while compared to referent groups of patients who had the risk factors and had not been treated with pioglitazone. Significant $P$-trends $(<0.01)$ were observed in all models, but $P$-interactions $(>0.05)$ were not significant, suggesting no interactions between pioglitazone and the risk factors.

\section{Discussion}

This study suggested a significantly lower risk of COPD in diabetes patients who had used pioglitazone in comparison to those who had not used pioglitazone (Table 2 and Figure 2). No interactions were observed between pioglitazone and major risk factors of COPD including pneumonia, pulmonary tuberculosis and tobacco abuse, respectively (Table 3).

Table 2 Incidence Rates of Chronic Obstructive Pulmonary Disease and Hazard Ratios by Pioglitazone Exposure

\begin{tabular}{|c|c|c|c|c|c|c|c|}
\hline $\begin{array}{l}\text { Pioglitazone } \\
\text { Use }\end{array}$ & $\begin{array}{l}\text { Incident Case } \\
\text { Number }\end{array}$ & $\begin{array}{l}\text { Cases } \\
\text { Followed }\end{array}$ & $\begin{array}{l}\text { Person- } \\
\text { Years }\end{array}$ & $\begin{array}{c}\text { Incidence Rate (per I00,000 } \\
\text { Person-Years) }\end{array}$ & $\begin{array}{c}\text { Hazard } \\
\text { Ratio }\end{array}$ & $\begin{array}{l}\text { 95\% Confidence } \\
\text { Interval }\end{array}$ & $P$ value \\
\hline Never users & 359 & 9487 & $24,179.46$ & I 484.73 & 1.000 & & \\
\hline Ever users & 295 & 9487 & $25,265.21$ & $1|67.6|$ & 0.778 & $(0.667-0.908)$ & 0.0014 \\
\hline \multicolumn{8}{|c|}{ Tertiles of cumulative duration of pioglitazone therapy (months) } \\
\hline Never users & 359 & 9487 & $24,179.46$ & 1484.73 & 1.000 & & \\
\hline$<11.0$ & 108 & 3076 & 8002.90 & $|349.5|$ & 0.904 & $(0.729-1.121)$ & 0.3580 \\
\hline $11.0-19.6$ & 92 & 3163 & 8430.32 & 1091.30 & 0.727 & $(0.578-0.914)$ & 0.0063 \\
\hline$>19.6$ & 95 & 3248 & 8831.99 & 1075.64 & 0.715 & $(0.570-0.896)$ & 0.0036 \\
\hline
\end{tabular}


Table 3 Joint Effects Between Pioglitazone and Risk Factors of Chronic Obstructive Pulmonary Disease

\begin{tabular}{|c|c|c|c|c|c|c|c|}
\hline Risk Factor/Pioglitazone Use & Incident Case Number & Cases Followed & Person-Years & $\begin{array}{c}\text { Incidence Rate (per 100,000 } \\
\text { Person-Years) }\end{array}$ & Hazard Ratio & 95\% Confidence Interval & $P$ value \\
\hline Pneumonia (+)/Pioglitazone (-) & 70 & 801 & 1950.68 & 3588.50 & 1.000 & & \\
\hline Pneumonia $(+) /$ Pioglitazone $(+)$ & 66 & 843 & 2168.69 & 3043.31 & 0.879 & $(0.626-1.232)$ & 0.4531 \\
\hline Pneumonia (-)/Pioglitazone (-) & 289 & 8686 & $22,228.79$ & 1300.12 & 0.482 & $(0.367-0.632)$ & $<0.0001$ \\
\hline Pneumonia (-)/Pioglitazone (+) & 229 & 8644 & $23,096.52$ & 991.49 & 0.363 & $(0.275-0.480)$ & $<0.0001$ \\
\hline$P$-trend & & & & & & & $<0.0001$ \\
\hline$P$-interaction & & & & & & & 0.4333 \\
\hline Pulmonary TB (+)/Pioglitazone (-) & 17 & 182 & 427.86 & 3973.29 & 1.000 & & \\
\hline Pulmonary TB (+)/Pioglitazone (+) & 14 & 178 & 460.32 & 3041.38 & 0.686 & $(0.337-1.395)$ & 0.2978 \\
\hline Pulmonary TB (-)/Pioglitazone (-) & 342 & 9305 & $23,751.61$ & 1439.90 & 0.504 & $(0.307-0.826)$ & 0.0065 \\
\hline Pulmonary TB (-)/Pioglitazone (+) & 281 & 9309 & $24,804.89$ & 1132.84 & 0.395 & $(0.240-0.649)$ & 0.0002 \\
\hline$P$-trend & & & & & & & $<0.0001$ \\
\hline$P$-interaction & & & & & & & 0.7185 \\
\hline Tobacco abuse $(+) /$ Pioglitazone (-) & 17 & 338 & 849.32 & 2001.59 & 1.000 & & \\
\hline Tobacco abuse $(+) /$ Pioglitazone $(+)$ & 12 & 325 & 879.70 & 1364.10 & 0.650 & $(0.310-1.364)$ & 0.2549 \\
\hline Tobacco abuse (-)/Pioglitazone (-) & 342 & 9149 & $23,330.14$ & $1465.9 \mid$ & $0.74 I$ & $(0.453-1.214)$ & 0.2343 \\
\hline Tobacco abuse (-)/Pioglitazone $(+)$ & 283 & 9162 & $24,385.51$ & 1160.53 & 0.582 & $(0.355-0.956)$ & 0.0325 \\
\hline$P$-trend & & & & & & & 0.0013 \\
\hline$P$-interaction & & & & & & & 0.6247 \\
\hline Any of the three $(+) /$ Pioglitazone $(-)$ & 96 & 1223 & 2998.79 & 3201.29 & 1.000 & & \\
\hline Any of the three $(+) /$ Pioglitazone $(+)$ & 84 & 1235 & 3227.92 & 2602.30 & 0.851 & $(0.634-1.142)$ & 0.2821 \\
\hline All of the three (-)/Pioglitazone (-) & 263 & 8264 & $21,180.67$ & 1241.70 & 0.467 & $(0.367-0.593)$ & $<0.0001$ \\
\hline All of the three $(-) /$ Pioglitazone $(+)$ & 211 & 8252 & $22,037.29$ & 957.47 & 0.355 & $(0.277-0.455)$ & $<0.0001$ \\
\hline$P$-trend & & & & & & & $<0.0001$ \\
\hline$P$-interaction & & & & & & & 0.5250 \\
\hline
\end{tabular}

Abbreviation: TB, tuberculosis. 
A previous human observational retrospective cohort study conducted in the US veterans suggested that TZDs-treated diabetes patients with COPD had a significantly lower risk of exacerbation of COPD than diabetes patients with COPD who had not been treated with TZDs. ${ }^{24}$ Another recent observational study conducted in Taiwan supported that the use of TZDs was associated with a reduced risk of acute exacerbation of COPD in patients with T2DM and COPD. ${ }^{29}$ The findings of these previous studies combined together with ours suggested that TZDs might be useful for reducing the risk of COPD and reducing the exacerbation of COPD in patients with T2DM. It is worthy to note that the benefit observed for COPD in the previous US study mainly reflected the use of rosiglitazone (97.1\% of the users of TZDs) ${ }^{24}$ the previous Taiwanese study did not differentiate pioglitazone from rosiglitazone ${ }^{29}$ and our present study investigated only pioglitazone. Therefore, it requires additional research to examine whether both pioglitazone and rosiglitazone may have similar effects on the prevention of new-onset COPD and on the prevention of acute exacerbation of COPD.

There are some possible explanations for the mechanisms of a reduced risk of COPD associated with pioglitazone use. PPAR $\gamma$ signaling is important in the normal development and repair of the lung. ${ }^{30}$ An animal study conducted in Sprague-Dawley rats that compared the efficacy of dexamethasone and pioglitazone showed that antenatal administration of pioglitazone can accelerate fetal lung maturation equally in both sexes, which could be superior to steroid that showed a sex-specific effect (more effective in female rats). ${ }^{31}$ In an animal model of COPD, both rosiglitazone and pioglitazone attenuated pulmonary inflammation by reducing tumor necrosis factor- $\alpha, \mathrm{CC}$ chemokine ligand-5 and airway neutrophilia. ${ }^{22}$ In another mouse model of smoke-induced emphysema, PPAR $\gamma$ expression was downregulated, which could be reversed by the treatment of ciglitazone, a PPAR $\gamma$ agonist. ${ }^{23}$ Pioglitazone can reduce sepsis-induced lung injury by reducing inflammation ${ }^{19}$ and potentiate the repair of lung injury by mesenchymal stem cells. ${ }^{20,21}$ Therefore, the mechanisms of a lower risk of COPD might be related to an upregulation and activation of PPAR $\gamma$ and the subsequent anti-inflammatory effects and injury repair capability of pioglitazone.

There are several clinical implications of the present study. First, because pioglitazone is minimally excreted by the kidney, does not cause hypoglycemia and may be preventive for cardiovascular diseases, ${ }^{10}$ especially ischemic stroke, ${ }^{11}$ it can be considered an option for patients who fail a successful treatment with metformin, especially for patients with kidney disease, ischemic stroke or at a high risk of COPD. Second, if not contraindicated and without remarkable side effects (such as heart failure), pioglitazone should better be continuously used for at least 11 months for a significant risk reduction of COPD (Table 2). Third, taking into account the potential, though not conclusive, risk of bladder cancer, ${ }^{13}$ the use of pioglitazone should be weighed for risks and benefits in patients with a history of bladder cancer.

The study has some merits. First, the generalization of the findings to the whole population can be more feasible because we used a nationwide database with a high coverage rate of $>99 \%$ of the Taiwan's population. Second, biases resulted from self-reporting can be significantly avoided by using pre-existing medical records. Third, because the copayment in our NHI healthcare system is very low and most cost can actually be waived if the patients have lowincome, are veterans or receive refills of drugs for chronic diseases, detection bias due to different socioeconomic status was less likely.

The study may also have some limitations. First, we did not have measurement data of some confounders, such as body height, body weight, nutritional status, lifestyle, dietary pattern, smoking, alcohol drinking, occupational and environmental exposure, family history and genetic parameters. Second, we did not have data of blood levels of glucose, lipid profiles and inflammatory biomarkers for analyses. Third, the lack of imaging studies or pulmonary function tests for the related diagnoses might have caused misdiagnosis in some patients. However, if the misclassification was not differential, the estimated hazard ratios were expected to biased toward the null. ${ }^{32}$ Fourth, oxidative stress and inflammation are associated with an increased mucus secretion in patients with COPD. Studies suggested that supplementation with antioxidants such as Nigella Sativa seed and seed oil consumption ${ }^{33}$ and probiotics/synbiotics ${ }^{34}$ may lower oxidative stress and inflammation. We did not have information on the use of these supplements in the database for in-depth analyses. Fifth, some polyphenolic compounds, ${ }^{35}$ magnesium ${ }^{36}$ and novel lipid-lowering drugs ${ }^{37}$ can affect the control of diabetes and lipid disorders. These may exert confounding effects but we did not have related information to assess the potential bias related to their use. Finally, patients with T2DM are characterized by multiple comorbidities and complications and therefore polypharmacy is the general condition. ${ }^{38}$ Except for pneumonia, pulmonary tuberculosis and 
tobacco abuse (Table 3), we did not investigate the interactions among various drugs or in the presence of some other complications or comorbidities.

In summary, this study conducted in the Taiwanese patients with T2DM suggests that pioglitazone may have a beneficial effect on the occurrence of COPD, especially when it has been used for $>11$ months. Interactions between pioglitazone and COPD risk factors for pneumonia, pulmonary tuberculosis and tobacco abuse do not exist. Further confirmation of the findings is warranted; but in patients with COPD risk factors, pioglitazone can be considered as a second-line therapy following metformin.

\section{Acknowledgments}

The author thanks Ms. Ting-Ting Chan for her excellent help in statistical analyses.

\section{Funding}

The study was partially supported by the Ministry of Science and Technology (MOST 103-2314-B-002-187-MY3) and the Yee Fong Charity Foundation.

\section{Disclosure}

The author declares no conflicts of interest.

\section{References}

1. Srivastava SP, Goodwin JE. Cancer biology and prevention in diabetes. Cells. 2020;9(6):1380. doi:10.3390/cells9061380

2. Daryabor G, Atashzar MR, Kabelitz D, Meri S, Kalantar K. The effects of type 2 diabetes mellitus on organ metabolism and the immune system. Front Immunol. 2020;11:1582. doi:10.3389/fimmu.2020.01582

3. Papazafiropoulou AK, Koros C, Melidonis A, Antonopoulos S. Diabetes and dementia - the two faces of Janus. Arch Med Sci Atheroscler Dis. 2020;5:e186-e197. doi:10.5114/amsad.2020.97433

4. Dalle S, Koppo K. Is inflammatory signaling involved in disease-related muscle wasting? Evidence from osteoarthritis, chronic obstructive pulmonary disease and type II diabetes. Exp Gerontol. 2020;137:110964. doi:10.1016/j.exger.2020.110964

5. Khateeb J, Fuchs E, Khamaisi M. Diabetes and lung disease: a neglected relationship. Rev Diabet Stud. 2019;15:1-15. doi:10.1900/RDS.2019.15.1

6. Diaconu C, Salmen T, Gaman MA, et al. SGLT2 inhibition in patients with type 2 diabetes and cardiovascular diseases: which are the benefits? Rom $J$ Mil Med. 2019;CXXII(1):16-21.

7. Tseng $\mathrm{CH}$, Tseng FH. Peroxisome proliferator-activated receptor agonists and bladder cancer: lessons from animal studies. $J$ Environ Sci Health C Environ Carcinog Ecotoxicol Rev. 2012;30(4):368-402. doi:10.1080/10590501.2012.735519

8. Nissen SE, Wolski K. Effect of rosiglitazone on the risk of myocardial infarction and death from cardiovascular causes. $N$ Engl $J$ Med. 2007;356 (24):2457-2471. doi:10.1056/NEJMoa072761

9. Tseng CH, Huang TS. Pioglitazone with sulfonylurea: glycemic and lipid effects in Taiwanese diabetic patients. Diabetes Res Clin Pract. 2005;70 (2): 193-194. doi:10.1016/j.diabres.2004.11.003

10. Dormandy JA, Charbonnel B, Eckland DJ, et al.; for PROactive investigators. Secondary prevention of macrovascular events in patients with type 2 diabetes in the PROactive Study (PROspective pioglitAzone Clinical Trial In macroVascular Events): a randomised controlled trial. Lancet. 2005;366(9493):1279-1289. doi:10.1016/S0140-6736(05)67528-9

11. Kernan WN, Viscoli CM, Furie KL, et al.; for IRIS Trial Investigators. Pioglitazone after ischemic stroke or transient ischemic attack. $N$ Engl $J$ Med. 2016;374(14):1321-1331. doi:10.1056/NEJMoa1506930

12. Lewis JD, Ferrara A, Peng T, et al. Risk of bladder cancer among diabetic patients treated with pioglitazone: interim report of a longitudinal cohort study. Diabetes Care. 2011;34(4):916-922. doi:10.2337/dc10-1068

13. Hampp C, Pippins J. Pioglitazone and bladder cancer: FDA's assessment. Pharmacoepidemiol Drug Saf. 2017;26(2):117-118. doi:10.1002/ pds.4154

14. Tseng CH. Diabetes and risk of bladder cancer: a study using the National Health Insurance database in Taiwan. Diabetologia. 2011;54 (8):2009-2015. doi:10.1007/s00125-011-2171-z

15. Tseng CH. Pioglitazone and bladder cancer: a population-based study of Taiwanese. Diabetes Care. 2012;35(2):278-280. doi:10.2337/dc11-1449

16. Tseng CH. Rosiglitazone is not associated with an increased risk of bladder cancer. Cancer Epidemiol. 2013;37(4):385-389. doi:10.1016/j. canep.2013.03.013

17. Tseng CH. Pioglitazone and lung cancer risk in Taiwanese patients with type 2 diabetes. Diabetes Metab. 2018;44(1):77-79. doi:10.1016/j. diabet.2017.05.011

18. Sekine Y, Katsura H, Koh E, Hiroshima K, Fujisawa T. Early detection of COPD is important for lung cancer surveillance. Eur Respir J. 2012;39 (5):1230-1240. doi:10.1183/09031936.00126011

19. Kutsukake M, Matsutani T, Tamura K, et al. Pioglitazone attenuates lung injury by modulating adipose inflammation. J Surg Res. 2014;189 (2):295-303. doi:10.1016/j.jss.2014.03.007

20. Hong Y, Kim YS, Hong SH, Oh YM. Therapeutic effects of adipose-derived stem cells pretreated with pioglitazone in an emphysema mouse model. Exp Mol Med. 2016;48(10):e266. doi:10.1038/emm.2016.93 
21. Park JS, Kim HK, Kang EY, Cho R, Oh YM. Potential therapeutic strategy in chronic obstructive pulmonary disease using pioglitazone-augmented Wharton's jelly-derived mesenchymal stem cells. Tuberc Respir Dis. 2019;82(2):158-165. doi:10.4046/trd.2018.0044

22. Lea S, Plumb J, Metcalfe H, et al. The effect of peroxisome proliferator-activated receptor- $\gamma$ ligands on in vitro and in vivo models of COPD. Eur Respir J. 2014;43(2):409-420. doi:10.1183/09031936.00187812

23. Shan M, You R, Yuan X, et al. Agonistic induction of PPAR $\gamma$ reverses cigarette smoke-induced emphysema. J Clin Invest. 2014;124(3):1371-1381. doi:10.1172/JCI70587

24. Rinne ST, Liu CF, Feemster LC, et al. Thiazolidinediones are associated with a reduced risk of COPD exacerbations. Int J Chron Obstruct Pulmon Dis. 2015;10:1591-1597. doi:10.2147/COPD.S82643

25. Parsons LS. Performing a 1:N case-control match on propensity score. Available from: http://www2.sas.com/proceedings/sugi29/165-29.pdf. Accessed December 8, 2021.

26. Tseng CH. Metformin decreases risk of tuberculosis infection in type 2 diabetes patients. J Clin Med. 2018;7(9):264. doi:10.3390/jcm7090264

27. Tseng CH. Metformin use is associated with a lower risk of osteoporosis/vertebral fracture in Taiwanese patients with type 2 diabetes mellitus. Eur J Endocrinol. 2021;184(2):299-310. doi:10.1530/EJE-20-0507

28. Austin PC, Stuart EA. Moving towards best practice when using inverse probability of treatment weighting (IPTW) using the propensity score to estimate causal treatment effects in observational studies. Stat Med. 2015;34(28):3661-3679. doi:10.1002/sim.6607

29. Chen KY, Wu SM, Tseng $\mathrm{CH}$, et al. Combination therapies with thiazolidinediones are associated with a lower risk of acute exacerbations in new-onset COPD patients with advanced diabetic mellitus: a cohort-based case-control study. BMC Pulm Med. 2021;21(1):141. doi:10.1186/ s12890-021-01505-7

30. Rehan VK, Torday JS. PPAR $\gamma$ Signaling mediates the evolution, development, homeostasis, and repair of the lung. PPAR Res. 2012;2012:289867. doi: $10.1155 / 2012 / 289867$

31. Lee C, Sakurai R, Shin E, Wang Y, Liu J, Rehan VK. Antenatal PPAR- $\gamma$ agonist pioglitazone stimulates fetal lung maturation equally in males and females. Am J Physiol Lung Cell Mol Physiol. 2020;319(3):L435-L443. doi:10.1152/ajplung.00376.2018

32. Kesmodel US. Information bias in epidemiological studies with a special focus on obstetrics and gynecology. Acta Obstet Gynecol Scand. $2018 ; 97$ (4):417-423. doi:10.1111/aogs.13330

33. Montazeri RS, Fatahi S, Sohouli MH, et al. The effect of nigella sativa on biomarkers of inflammation and oxidative stress: a systematic review and meta-analysis of randomized controlled trials. J Food Biochem. 2021;45(4):e13625. doi:10.1111/jfbc.13625

34. Pourrajab B, Fatahi S, Sohouli MH, Găman MA, Shidfar F. The effects of probiotic/synbiotic supplementation compared to placebo on biomarkers of oxidative stress in adults: a systematic review and meta-analysis of randomized controlled trials. Crit Rev Food Sci Nutr. $2022 ; 62: 490-507$. doi:10.1080/10408398.2020.1821166

35. Egbuna C, Awuchi CG, Kushwaha G, et al. Bioactive compounds effective against type 2 diabetes mellitus: a systematic review. Curr Top Med Chem. 2021;21(12):1067-1095. doi:10.2174/1568026621666210509161059

36. Găman MA, Dobrică EC, Cozma MA, et al. Crosstalk of magnesium and serum lipids in dyslipidemia and associated disorders: a systematic review. Nutrients. 2021;13(5):1411. doi:10.3390/nu13051411

37. Crismaru I, Pantea Stoian A, Bratu OG, et al. Low-density lipoprotein cholesterol lowering treatment: the current approach. Lipids Health Dis. 2020;19(1):85. doi:10.1186/s12944-020-01275-x

38. Dobrică EC, Găman MA, Cozma MA, Bratu OG, Pantea Stoian A, Diaconu CC. Polypharmacy in type 2 diabetes mellitus: insights from an internal medicine department. Medicina. 2019;55(8):436. doi:10.3390/medicina55080436

International Journal of Chronic Obstructive Pulmonary Disease

Dovepress

\section{Publish your work in this journal}

The International Journal of COPD is an international, peer-reviewed journal of therapeutics and pharmacology focusing on concise rapid reporting of clinical studies and reviews in COPD. Special focus is given to the pathophysiological processes underlying the disease, intervention programs, patient focused education, and self management protocols. This journal is indexed on PubMed Central, MedLine and CAS. The manuscript management system is completely online and includes a very quick and fair peer-review system, which is all easy to use. Visit http://www. dovepress.com/testimonials.php to read real quotes from published authors.

Submit your manuscript here: https://www.dovepress.com/international-journal-of-chronic-obstructive-pulmonary-disease-journal 